\title{
The scattering mean free path of cosmic ray particles in isotropic damped plasma wave turbulence
}

\author{
M. Vukcevic ${ }^{1,2}$ \\ ${ }^{1}$ Institut für Theoretische Physik, Lehrstuhl IV: Weltraum- und Astrophysik, Ruhr-Universität Bochum, 44780 Bochum, Germany \\ e-mail: vuk.mira@gmail.com \\ 2 University of Defence, Military Academy, Pavla Jurisica Sturma 33, 11000 Belgrade, Serbia
}

Received 30 January 2013 / Accepted 3 May 2013

\section{ABSTRACT}

\begin{abstract}
An analytical expression for the mean free path of single-charged cosmic ray particles, especially for positrons, is derived in isotropic plasma wave turbulence, where the crucial scattering of cosmic ray particles with small pitch-angle cosines is caused by resonant cyclotron interactions with oblique magnetosonic waves. In this calculation, viscous damping effects are included, which results in broadening of the resonance function. It is demonstrated that including resonance function broadening ensures a finite mean free path for cosmic ray energies, for which previously reported types of turbulence predicted an infinitely large mean free path.
\end{abstract}

Key words. scattering

\section{Introduction}

A Galactic bulge-to-disk ratio of the luminosity of diffuse $511 \mathrm{keV}$ positron annihilation radiation is, as measured by INTEGRAL, four times larger than a stellar bulge to disk ratio of the Galactic supernovae (SNe). SNe are thought to be the principal source of the annihilating positrons. This large discrepancy has started a search for new sources. It has been shown that the measured $511 \mathrm{keV}$ luminosity ratio can be understood well in the context of a Galactic SN origin when the differential propagation of these $\mathrm{MeV}$ positrons in the various phases of the interstellar medium is taken into consideration (Higdon et al. 2009), since these relativistic positrons must first slow down to energies less than $10 \mathrm{eV}$ before they can annihilate.

A large number of potential sources have been proposed over the years: cosmic ray interaction with the interstellar medium (Ramaty et al. 1970), pulsars (Sturrock 1971), radioactive nuclei produced in SN (Clayton 1973), compact objects (Ramaty \& Lingelfelter 1979), dark matter (Boehm 2004), and microquasars (Guessoum et al. 2006). Some of these theories either are problematic or have a wide range of uncertainties. For example, the predicted distributions of positrons from radionuclei synthesized in SN are only marginally compatible with observations (Milne et al. 2002). However, the recent mapping of the Galaxy at $511 \mathrm{KeV}$ (Knödlseder et al. 2005) has placed severe constraints on the possible positron sources.

On the other hand, the positron excess in Galactic cosmic ray positrons above $10 \mathrm{GeV}$ has been confirmed by PAMELA (Adriani et al. 2009). The most recent confirmation of the PAMELA result on positrons in the Galaxy cosmic ray spectrum has just been published (Aguilar et al. 2013). There are several astrophysical explanations for possible sources of these positrons, such as dark matter annihilation (Hooper et al. 2009) or decay (Arvanitaki et al. 2009). According to a recent investigation (Pohl \& Eichler 2009), Fermi measurements of the high-latitude $\gamma$-ray background (Abdo et al. 2009) constrain a decaying-dark-matter origin for the $\mathrm{GeV}$ Galactic positron anomaly measured by PAMELA.

To quantify scattering mean free paths for $\mathrm{MeV}$ interstellar positrons, an analytic approximation has been used (Teufel $\&$ Schlickeiser 2002) for slab-like dynamical turbulence, which predict that is a mean free path in $\mathrm{MeV}$ energies proportional to $r^{1 / 3}$, where $r$ is defined as particle rigidity. This model for $\mathrm{GeV}$ particles predicts an infinitely large mean free path. The other slab plasma-wave turbulence model (Schlickeiser et al. 2010), which is more plausible when compared to the dynamical one, predicts an infinite positron scattering mean free path at $\mathrm{MeV}$ energies, while it has finite values proportional to $r^{1 / 3}$ for $\mathrm{GeV}$ energies.

Here, we propose a damped plasma wave turbulence model, which can assure a finite mean free path for $\mathrm{MeV}$ and $\mathrm{GeV}$ positrons, and compare our result with results of previously mentioned models.

A key parameter for the cosmic ray transport is the parallel spatial diffusion coeffcient $\kappa=v \lambda / 3$, which is conventionally expressed in terms of the mean free path $\lambda$ along the background magnetic field and the particle speed $v$. In many studies, the parallel mean free path also controls the perpendicular spatial diffusion coefficient $\kappa_{\perp}=\alpha \kappa_{\|}$, which is assumed to be proportional to $\kappa_{\|}$due to the lack of a rigorous theory of perpendicular diffusion. When discussing the ratio of perpendicular and parallel mean free path, it has been published from proton observations that $0.02<\lambda_{\perp} / \lambda_{\|}<0.083$ over the rigidity range of $0.5 M V<R<5 G V$ (Palmer 1982), which assures parallel mean free path values to be high enough that we can neglect the perpendicular component. Hereafter, we only consider parallel mean free path.

The scattering mean free path is the result of resonant particle-wave interactions of cosmic ray particles with the turbulent component of cosmic magnetic fields, and thus depends on the nature and geometry of cosmic turbulent magnetic fields (Schlickeiser 2002). Many observations of plasma turbulence 
in the solar wind indicate that the turbulent magnetic field is a mixture of slab (i.e., parallel to the ordered background magnetic field) waves, a dominating 2D component (Bieber et al. 1996) with a negligible contribution to particle scattering (Shalchi $\&$ Schlickeiser 2004), and obliquely propagating magnetosonic waves that reveal through electron density fluctuations because of their compressive nature. In the static magnetosonic limit, the scattering rate from the 2D component vanishes (Shalchi \& Schlickeiser 2004).

For small amplitude waves, an appreciable interaction between a wave and a particle arises only when the particle is moving at nearly the parallel wave phase speed. The interaction is resonant and the subsequent physics is close to that of Fermi acceleration: particles with $v_{\|}$slightly greater than $\omega / k_{\|}$suffer a trailing collision with a wave compression and slow down, while particles with $v_{\|}$slightly less than $\omega / k_{\|}$are stuck by a compression and speed up. This process could be called resonant Fermi acceleration, but the usual term is transit-time acceleration or transit-time damping (TTD). The name is given because the resonance condition can be rewritten as $\lambda_{\|} / v_{\|} \approx t$, where $t$ is the wave period and $\lambda_{\|}=2 \pi / k \eta$ is the parallel wavelength. That means a wave and a particle will interact strongly when the particle transit time across the wave compression is approximately equal to the period.

Schlickeiser \& Miller (1998) and Schlickeiser \& Vainio (1999) have investigated the quasilinear interactions of charged particles with Alfven and magnetosonic plasma modes. A cosmic ray particle of given velocity $v$, Lorentz factor $\gamma=(1-$ $\left.\left(v^{2} / c^{2}\right)\right)^{-1 / 2}$, pitch angle cosine $\mu=v_{\|} / v$, mass $m$, the speed of light $c$, charge $q_{i}=e\left|Z_{i}\right| Q$ with $Q=\operatorname{sgn}\left(Z_{i}\right)$, and gyrofrequency $\Omega_{\mathrm{c}}=Q \Omega$ with $\Omega=q_{i} B_{0} /(m c \gamma)$ interacts with waves whose wavenumber $k$, cosine of propagation angle $\eta=k_{\|} / k$, and real frequency $f$ obey the resonance condition $f(k)=v \mu k \eta+n \Omega_{\mathrm{c}}$ for entire $n \in[-\infty, \infty]$. For $\operatorname{slab}(\eta=1)$ Alfven waves, only gyroresonant interactions with $n=1$ are possible. In contrast, the $n=0$ resonance for obliquely propagating fast magnetosonic waves, which do not include gyroresonance interactions $(n \neq 1)$, is possible due to their compressive magnetic field component. The $n=0$ interactions are referred to as transit-time damping (TTD). Using $n=0$ the resonance condition requires the parallel cosmic ray velocity $v \mu=f /(k \eta) f / k \simeq V_{\mathrm{A}}$ to be greater than the Pfinite phase velocity of magnetosonic waves, which is given by the Alfven speed. TTD interactions therefore only occur for cosmic ray particles with pitch-angle cosines $\mu>\left|V_{\mathrm{A}} / v\right|=\epsilon$. At these pitch angle cosines, TTD interactions provide most of the particle scattering.

Thus, the crucial scattering in small $\mu$ is provided for energetic particles by only gyroresonances with slab plasma waves and oblique magnetosonic waves (Schlickeiser et al. 2010):

$\lambda=\frac{3 v}{8} \int_{-\epsilon}^{\epsilon} \mathrm{d} \mu \frac{\left(1-\mu^{2}\right)}{D_{\mu \mu}^{\mathrm{slab}}(\mu)+D_{\mu \mu}^{\mathrm{g}-\mathrm{ms}}(\mu)} \simeq \frac{3 V_{\mathrm{A}}}{4\left[D_{\mu \mu}^{\mathrm{slab}}(0)+D_{\mu \mu}^{\mathrm{g}-\mathrm{ms}}(0)\right]}$.

According to Schlickeiser \& Miller (1998), the magnetosonic contribution $D_{\mu \mu}^{\mathrm{g}-\mathrm{ms}}(0) \simeq \epsilon D_{\mu \mu}^{\mathrm{slab}}(0)$ for energetic particles $(\epsilon \ll 1)$ is much smaller than the slab contribution for similar turbulence power spectra of slab and magnetosonic waves. However, this latter reduction is not justied for cosmic ray positrons with Lorentz factors $\gamma<m_{\mathrm{p}} / m_{\mathrm{e}}=1836$. These positrons no LH polarized slab waves to find resonantly interact with, so that $D_{\mu \mu}^{\text {slab }}(0)->0$. In this case, the contribution from gyroresonant interactions with magnetosonic waves provides small but finite scattering (Schlickeiser et al. 2010).
It is the purpose of this work to involve oblique magenetosonic waves and quantitatively investigate the influence of wave damping on the quasilinear scattering mean free paths of cosmic rays, especially for positrons with Lorentz factors $\gamma<m_{\mathrm{p}} / m_{\mathrm{e}}=1836$. In a plasma in rough equipartition of kinetic and magnetic pressure, oblique magnetosonic waves are expected to be overdamped by Landau absorption (Fedorenko 1992). Thus, the second-order Fermi acceleration using oblique fast magnetosonic waves cannot work in a plasma in rough equipartiton (Achterberg 1979). It is possible to overcome this difficulty either by considering that this mechanism works, in the interstellar medium with small $\beta$ (relatively cold medium) or by including the finite wave cascading that exists in the interstellar medium. Due to the large amplitude of interstellar turbulence, there is no doubt that wave cascading exists and will then transfer this spectral energy to higher wavenumbers. One property of the diffusion equation for isotropic turbulence (Eq. (11.3.6), Schlickeiser 2002) is that it yields finite positive wave spectral energy densities at all wavenumbers $k$, even if the net damping rate is negative in some wavenumber intervals.

We show then that the inclusion of resonance broadening caused by wave damping in the resonance function guarantees that the transit-time damping contribution holds at small pitch angle cosines $\mu \leq\left|V_{\mathrm{a}} / v\right|$, unlike the case of neglible wave damping (Vukcevic \& Schlickeiser 2007). We expect that TTD makes an overwhelming contribution to particle scattering because the cosmic ray particle interacts with the whole wave spectrum in this interaction. This contrasts gyroresonances that single out individual resonant wave numbers (Schlickeiser 2003). However, quantitative analysis of gyroresonance contribution in damped plasma wave turbulence will be studied in a separate, forthcoming investigation. Therefore we only consider the TTD-contribution to particle scattering in the following and assume $n=0$ both in the resonance function and in the calculation of the Fokker-Planck coefficients.

In Sect. 2, we present the general plasma wave turbulence and relevant magnetohydrodynamic plasma modes. In Sect. 3, we calculate the Fokker-Planck coefficient for fast mode waves and discuss relevant domains for application to cosmic ray positrons. In Sect. 4, we derived the Fokker-Planck coefficient for slow mode waves. The analytical expressions of a mean free path for fast and slow plasma waves and the comparison with different previously reported turbulence models are given in Sect. 5 . The summary and conclusion are presented in the last section.

\section{Plasma wave turbulence}

According to our discussion in the paragraph above and in the two paragraphs after Eq. (1), the relevant mean free path for the positrons with Lorentz factor $\gamma<m_{\mathrm{p}} / m_{\mathrm{e}}=1836$ in small pitchangle cosine becomes

$$
\lambda \simeq \frac{3 V_{\mathrm{A}}}{4 D_{\mu \mu}^{\mathrm{TTD}}(0)}
$$

Hereafter, we omit TTD notation and keep only notation for relevant magnetosonic waves. The Fokker-Planck coefficient $D_{\mu \mu}$ is computed by employing the Kubo formula (Kubo 1957),

$D_{\mu \mu}=\int_{0}^{\infty} \mathrm{d} t\langle\dot{\mu}(t) \dot{\mu}(0)\rangle$. 
The pitch-angle variation $\dot{\mu}(t)$ is obtained from the Newton-Lorentz equation:

$$
\begin{aligned}
\dot{\mu} & =\frac{\Omega \sqrt{1-\mu^{2}}}{B_{0}}\left[\frac{c}{v} \sqrt{1-\mu^{2}} \delta E_{\|}+\frac{\mathrm{i}}{\sqrt{2}}\left[\mathrm{e}^{\mathrm{i} \phi}\left(\delta B_{r}+\mathrm{i} \mu \frac{c}{v} \delta E_{r}\right)\right.\right. \\
& \left.\left.-\mathrm{e}^{-\mathrm{i} \phi}\left(\delta B_{l}-\mathrm{i} \mu \frac{c}{v} \delta E_{l}\right)\right]\right] .
\end{aligned}
$$

In these equations, we use pitch-angle cosine $\mu$, the particle speed $v$, the gyrophase $\phi$, the cosmic ray particle gyrofrequency in the background field $B_{0}$ and the turbulent fields $\delta B_{l, r}$ and $\delta E_{l, r}$, which are related to the left-handed and right-handed polarized field components. The term $\delta E_{\|}$is parallel component relative to the background magnetic field.

The simplest method to calculate $D_{\mu \mu}$ is the application of perturbation theory (Jokipii 1966). In this case, we have applied a quasilinear approximation for a fluctuating electric and magnetic field, a quasistationary turbulence condition, and the existence of a finite correlation time $t_{\mathrm{c}}$. The last two assumptions guarantee a diffusive behavior of transport (Shalchi \& Schlickeiser 2004). The assumption of homogeneous turbulence will imply that the turbulence fields at different wavevectors are uncorrelated.

Next, we define the properties of the plasma turbulence that will be considered. We follow the approach for the electromagnetic turbulence that represents the Fourier transforms of the magnetic and electric field fluctuations as a superposition of $N$ individual weakly damped plasma modes of frequencies:

$$
\omega=\omega_{j}(\boldsymbol{k})=\omega_{R, j}(\boldsymbol{k})-i \gamma_{j}(\boldsymbol{k}),
$$

where $j=1, \ldots N$, which can have both the real and imaginary parts with $\left|\gamma_{j}\right| \ll\left|\omega_{R, j}\right|$, so that

$$
[\mathbf{B}(\boldsymbol{k}, t), \mathbf{E}(\boldsymbol{k}, t)]=\sum_{j=1}^{N}\left[\mathbf{B}^{j}(\boldsymbol{k}), \mathbf{E}^{j}(\boldsymbol{k})\right] \mathrm{e}^{-\mathrm{i} \omega_{j} t} .
$$

Damping of the waves is counted with a positive $\gamma^{j}>0$.

In the case we consider here, the time integration of the Fokker-Planck coefficient $D_{\mu \mu}$ yields the Lorentzian resonance function:

$$
\begin{aligned}
\mathcal{R}_{j}\left(\gamma_{j}\right) & =\int_{0}^{\infty} \mathrm{d} u \mathrm{e}^{-\mathrm{i}\left(k_{\|} \|_{\|}+\omega_{R, j}+n \Omega\right) u-\gamma_{j} u} \\
& =\frac{\gamma_{j}(\boldsymbol{k})}{\left.\gamma_{j}^{2}(\boldsymbol{k})+\left[k_{\|} v_{\|}+\omega_{R, j}(\boldsymbol{k})+n \Omega\right)\right]^{2}} .
\end{aligned}
$$

The detailed derivation of the Fokker-Planck coefficient for this case (vanishing magnetic helicity and isotropic turbulence) is performed in the PhD Thesis of Vukcevic (2007), which contrasts the case of negligible damping $\gamma->0$ (Schlickeiser 2001) in which the use of the $\delta$-function representation

$$
\lim _{\gamma \rightarrow 0} \frac{\gamma}{\gamma^{2}+\xi^{2}}=\pi \delta(\xi)
$$

reduces the resonance function (7) to sharp $\delta$-functions,

$\mathbf{R}^{j}(\gamma=0)=\pi \delta\left(k_{\|} v_{\|}+\omega_{R, j}+n \Omega\right)$.

Next, it is necessary to specify the geometry of the plasma wave turbulence itself through the correlation tensors, which will be adopted throughout this work in the form (Mattheus \& Smith 1981) of

$$
P_{\alpha \beta}^{j}(\boldsymbol{k})=\frac{g_{i}^{j}(\boldsymbol{k})}{\boldsymbol{k}^{2}}\left[\delta_{\alpha \beta}-\frac{\boldsymbol{k}_{\alpha} k_{\beta}}{k^{2}}+\mathrm{i} \sigma(\boldsymbol{k}) \epsilon_{\alpha \beta \lambda} \frac{k_{\lambda}}{k}\right],
$$

where $\sigma(\boldsymbol{k})$ is the magnetic helicity and the function $g(\boldsymbol{k})$ determines different turbulence geometries. This was to illustrate the result, although this model is not in accord with the known polarization properties of fast-mode waves at oblique angles.

\subsection{Relevant magnetohydrodynamic plasma modes}

It has been already emphasized that there is no TTD for shear Alfven waves (Teufel et al. 2003) in the case of negligible damping, and the gyroresonant interactions provided by shear Alfven waves is small compared to the same contribution provided by fast magnetosonic waves (Vukcevic \& Schlickeiser 2007). As a consequence, we consider only fast and slow magnetosonic waves.

\subsubsection{Fast magnetosonic plasma modes}

For the fast plasma modes, we use a simplified dispersion relation,

$\omega_{\mathrm{R}} \simeq j k V_{\mathrm{A}}$

which is relevant at wavenumbers $k_{\mathrm{c}} \ll k \ll \xi k_{\mathrm{c}}$, where $k_{\mathrm{c}}=\omega_{p, i} / c$ is the inverse ion skin length, and $\xi=$ $\sqrt{m_{\mathrm{p}} / m_{\mathrm{e}}}=43$.

In the dispersion equation, forward $(j=1)$ and backward $(j=-1)$ moving fast mode waves are described. The associated electric field and magnetic field polarizations are (Dogan et al. 2006)

$\delta E_{\mathrm{L}}=-\delta E_{\mathrm{R}}, \quad \delta E_{\|}=0, \quad \delta B_{\mathrm{L}}=\delta B_{\mathrm{R}}, \quad \delta B_{\|} \neq 0$.

\subsubsection{Slow magnetosonic plasma modes}

The dispersion relation for slow magnetosonic waves in low- $\beta$ plasma reads (Dogan et al. 2006)

$\omega_{\mathrm{R}}^{2} \simeq k^{2} V_{\mathrm{A}}^{2}\left(\frac{\eta^{2} \beta}{1+\beta}+\frac{\eta^{4} \beta^{2}}{(1+\beta)^{3}}\right)$

with $\eta=\cos \theta$ and $\beta$ as the ratio of thermal and magnetic pressure. In the last equation, we neglect the second term in brackets in the first approximation, since it is one order smaller than the first term. The associated electric field and magnetic field polarizations are

$\delta E_{\mathrm{L}}=-\delta E_{\mathrm{R}}, \quad \delta E_{\|}=0, \delta B_{\mathrm{L}}=\delta B_{\mathrm{R}}, \quad \delta B_{\|} \neq 0$.

Consideration of the dispersion relation in high frequencies could change the result, since we expect nondispersive effects in that range. The polarization of the waves in that domain could also affect the transport coefficients through the correlation tensors. However, a detailed inspection of the dispersion relation in the high-frequency domain is needed to draw a quantitative conclusion, and it will be discussed in a separate investigation.

\subsection{Damping rate}

The damping of magnetosonic waves is caused by both collisionless Landau damping and collisional viscous damping and by Joule damping and ion-neutral friction. The dominant contribution is provided by viscous damping with the rate calculated 
for plasma parameters of the diffuse intercloud medium (Spanier \& Schlickeiser 2005)

$$
\begin{aligned}
\gamma_{\mathrm{F}} & =\frac{1}{12} \beta V_{\mathrm{A}}^{2} \tau_{i} k^{2}\left[\sin ^{2} \theta+5 \times 10^{-9} \cos ^{2} \theta\right] \\
& =2.9 \times 10^{5} \beta V_{\mathrm{A}}^{2} k^{2}\left[\sin ^{2} \theta+5 \times 10^{-9} \cos ^{2} \theta\right]
\end{aligned}
$$

in terms of the ion-ion collisional time $\tau_{i}=3.5 \times 10^{6} \mathrm{~s}$. Except at very small propagation angles, the second term in Eq. (15) is negligible, and we infer

$\gamma_{\mathrm{F}} \simeq 2.9 \times 10^{5} \beta V_{\mathrm{A}}^{2} k^{2} \sin ^{2} \theta=\alpha_{\mathrm{F}} k^{2} \sin ^{2} \theta$,

where $\alpha_{\mathrm{F}}=\frac{1}{12} \beta V_{\mathrm{A}}^{2} \tau_{i}$.

\section{Fast mode waves}

With Eqs. (11) and (16), the resonance function (7) for forward and backward moving fast mode waves becomes

$\mathcal{R}_{\mathrm{F}}^{j}(n)=\frac{\alpha_{\mathrm{F}} k^{2} \sin ^{2} \theta}{\left.\left(\alpha_{\mathrm{F}} k^{2} \sin ^{2} \theta\right)^{2}+\left[k v \mu \cos \theta+j V_{\mathrm{A}} k+n \Omega\right)\right]^{2}}$,

which describes both gyroresonant $(n \neq 0)$ and transit-time damping $(n=0)$ wave-particle interactions.

The non-vanishing parallel magnetic field component $B_{\|} \neq 0$ (see Eq. (12)) of fast mode waves allows TTD interactions with $n=0$, so that we procede with $n=0$. The resonance function (17) becomes

$$
\begin{aligned}
\mathcal{R}_{\mathrm{F}}^{j}(0) & =\frac{\alpha_{\mathrm{F}} k^{2} \sin ^{2} \theta}{\left(\alpha_{\mathrm{F}} k^{2} \sin ^{2} \theta\right)^{2}+\left[k v \mu \cos \theta+j V_{\mathrm{A}} k\right]^{2}} \\
& =\frac{\alpha_{\mathrm{F}}\left(1-\eta^{2}\right)}{\left(\alpha_{\mathrm{F}} k\left(1-\eta^{2}\right)\right)^{2}+\left[v \mu \eta+j V_{\mathrm{A}}\right]^{2}} .
\end{aligned}
$$

Throughout this work, we consider isotropic turbulence $g^{j}(\boldsymbol{k})=$ $g^{j}(k)$. Modifications due to different turbulence geometries are possible and will be the subject of further analysis (in particular anisotropic turbulence).

For energetic cosmic ray particles with $v \gg V_{\mathrm{A}}$, the pitch-angle Fokker-Planck coefficient then simplifies as

$D_{\mu \mu}^{\mathrm{F}} \simeq \frac{\Omega^{2}}{4 B_{0}^{2}}\left(1-\mu^{2}\right) \sum_{j= \pm 1} \int_{-\infty}^{\infty} \mathrm{d} k \int_{-1}^{1} \mathrm{~d} \eta \mathcal{R}_{F}^{j}(0) g^{j}(k) J_{1}^{2}(W)\left(1+\eta^{2}\right)$,

where $J_{1}(W)$ is a Bessel function with the argument $W=$ $\frac{v}{|\Omega|} \cdot k_{\perp} \sqrt{1-\mu^{2}}=R_{\mathrm{L}} \cdot k_{\perp} \sqrt{1-\mu^{2}}$ that involves the cosmic ray Larmor radius $R_{\mathrm{L}}=v /|\Omega|$.

We further simplify Eq. (19) by assuming equal intensity of forward and backward waves (a vanishing cross helicity of each plasma mode):

$g^{+}(k)=g^{-}(k)=\frac{1}{2} g_{\text {tot }}(k)$,

which reads as

$D_{\mu \mu}^{\mathrm{F}} \simeq \frac{\Omega^{2}}{8 B_{0}^{2}}\left(1-\mu^{2}\right) \sum_{j= \pm 1} \int_{-\infty}^{\infty} \mathrm{d} k \int_{-1}^{1} \mathrm{~d} \eta \mathcal{R}_{F}^{j}(0) g_{\mathrm{tot}}^{j}(k) J_{1}^{2}(W)\left(1+\eta^{2}\right)$.

To illustrate our results, we adopt a Kolmogorov-type power law dependence on $g^{j}(k)$ above and below some minimum and maximum wavenumber $k_{\min }$ and $k_{\max }$, respectively

$g_{\text {tot }}(k)=g_{\text {tot }} k^{-q}$ for $k_{\min }<k<k_{\max }$.

The magnetic energy density in wave component $j$ is given by

$\left(\delta B_{j}\right)^{2}=\int_{0}^{\infty} \mathrm{d} k g^{j}(k)$

which implies

$g_{\mathrm{tot}}=(q-1)(\delta B)^{2} /\left(k_{\min }^{1-q}-k_{\max }^{1-q}\right) \simeq(q-1)(\delta B)^{2} k_{\min }^{q-1}$

for $k_{\max } \gg k_{\min }$.

With Eqs. (22), (23), (24) the pitch-angle Fokker-Planck coefficient $D_{\mu \mu}^{\mathrm{F}}$ reads as

$D_{\mu \mu}^{\mathrm{F}} \simeq \frac{\Omega^{2}}{4 B_{0}^{2}}(q-1)(\delta B)^{2} k_{\min }^{q-1}\left(1-\mu^{2}\right) \int_{k_{\min }}^{k_{\max }} \mathrm{d} k k^{-q}$

$\int_{-1}^{1} \mathrm{~d} \eta \mathcal{R}_{F}(0) J_{1}^{2}(W)\left(1+\eta^{2}\right)$

Now, we must approximate the resonance function (Eq. (18)). In doing this, we consider two cases:

a) $\eta<\eta_{\mathrm{c}}$

b) $\eta>\eta_{\mathrm{c}}$

where $\epsilon=V_{\mathrm{A}} / v$ and $\eta_{\mathrm{c}}=\epsilon / \mu$. The parameter $\eta_{\mathrm{c}}$ divides the integration domain with respect to $\eta$, in which either $V_{\mathrm{A}}, v \mu \eta$ or both values are relevant. By using $D_{\mu \mu}^{\mathrm{F}}(-\mu)=D_{\mu \mu}^{\mathrm{F}}(\mu)$ and the substitution $s=R_{\mathrm{L}} k \sqrt{1-\mu^{2}}$ and $R_{\mathrm{L}}=v /|\Omega|$, we derive

$D_{\mu \mu}^{\mathrm{F}} \simeq \alpha_{\mathrm{F}}(q-1)\left(k_{\min } R_{\mathrm{L}}\right)^{q-1}\left(\frac{\delta B}{B_{0}}\right)^{2}\left(1-\mu^{2}\right)^{\frac{q+1}{2}} \int_{k_{\min } R_{\mathrm{L}} \sqrt{1-\mu^{2}}}^{\infty} \mathrm{d} s s^{-q}$

$\times\left[\int_{0}^{\min (1, \epsilon / \mu)} \mathrm{d} \eta\left(1-\eta^{4}\right) J_{1}^{2}\left(s \sqrt{1-\eta^{2}}\right) \frac{1}{\frac{\alpha_{\mathrm{F}}^{2}\left(1-\eta^{2}\right)^{2} s^{2}}{R_{\mathrm{L}}^{2}\left(1-\mu^{2}\right)}+V_{\mathrm{A}}^{2}}\right.$

$\left.+\int_{\min (1, \epsilon / \mu)}^{1} \mathrm{~d} \eta\left(1-\eta^{4}\right) J_{1}^{2}\left(s \sqrt{1-\eta^{2}}\right) \frac{1}{\frac{\alpha_{\mathrm{F}}^{2}\left(1-\eta^{2}\right)^{2} s^{2}}{R_{\mathrm{L}}^{2}\left(1-\mu^{2}\right)}+(v \mu \eta)^{2}}\right]$

\subsection{High values of $\mu>\epsilon$}

For large pitch-angles $\mu>\epsilon$ we obtain

$D_{\mu \mu}^{\mathrm{F}}(\mu>\epsilon) \simeq \frac{(q-1)}{\alpha_{\mathrm{F}}}\left(k_{\min } R_{\mathrm{L}}\right)^{q-1}\left(\frac{\delta B}{B_{0}}\right)^{2}\left(1-\mu^{2}\right)^{\frac{q+3}{2}}$

$\times \int_{k_{\min } R_{\mathrm{L}} \sqrt{1-\mu^{2}}}^{\infty} \mathrm{d} s s^{-q}\left[\int_{0}^{\epsilon / \mu} \mathrm{d} \eta\left(1-\eta^{4}\right) J_{1}^{2}\left(s \sqrt{1-\eta^{2}}\right)\right.$

$+\frac{1}{\left(1-\eta^{2}\right)^{2} s^{2}+\frac{R_{\mathrm{L}}^{2}\left(1-\mu^{2}\right) V_{\mathrm{A}}^{2}}{\alpha_{\mathrm{F}}^{2}}}+\int_{\epsilon / \mu}^{1} \mathrm{~d} \eta\left(1-\eta^{4}\right) J_{1}^{2}\left(s \sqrt{1-\eta^{2}}\right)$

$\left.\times \frac{1}{\left(1-\eta^{2}\right)^{2} s^{2}+\frac{R_{\mathrm{L}}^{2}\left(1-\mu^{2}\right)(v \mu \eta)^{2}}{\alpha_{\mathrm{F}}^{2}}}\right]$. 
M. Vukcevic: The scattering mean free path of cosmic ray particles in isotropic damped plasma wave turbulence

\subsection{Small values $\mu<\epsilon$}

This case is important when treating damped waves. For the small pitch-angles $\mu<\epsilon$, we obtain

$$
\begin{aligned}
& D_{\mu \mu}^{\mathrm{F}}(\mu<\epsilon) \simeq \alpha_{\mathrm{F}}(q-1)\left(k_{\min } R_{\mathrm{L}}\right)^{q-1}\left(\frac{\delta B}{B_{0}}\right)^{2}\left(1-\mu^{2}\right)^{\frac{q+1}{2}} \\
& \times \int_{k_{\min } R_{\mathrm{L}} \sqrt{1-\mu^{2}}}^{\infty} \mathrm{d} s s^{-q}\left[\int_{0}^{1} \mathrm{~d} \eta\left(1-\eta^{4}\right) J_{1}^{2}\left(s \sqrt{1-\eta^{2}}\right)\right. \\
& \left.\times \frac{1}{\frac{\alpha_{\mathrm{F}}^{2}\left(1-\eta^{2}\right)^{2} s^{2}}{R_{\mathrm{L}}^{2}\left(1-\mu^{2}\right)}+V_{\mathrm{A}}^{2}}\right] .
\end{aligned}
$$

We have already discussed that inclusion of resonance broadening due to wave damping in the resonance function guarantees dominance of transit-time damping. The main contribution of wave damping comes exactly in the region $|\mu|<\epsilon$ that is relevant for deriving the spatial diffusion coefficient and related mean free path, both of which are given by the average over $\mu$ of the inverse of $D_{\mu \mu}$. Therefore, we can further consider only the case $D_{\mu \mu}(\mu=0)$, which simplifies the analysis enormously and reads as

$$
\begin{aligned}
& D_{\mu \mu}^{\mathrm{F}}(\mu=0) \simeq \frac{\Omega^{2}(q-1) R_{\mathrm{L}}^{2}}{4 \alpha_{\mathrm{F}}}\left(k_{\min } R_{\mathrm{L}}\right)^{q-1}\left(\frac{\delta B}{B_{0}}\right)^{2} \int_{k_{\min } R_{\mathrm{L}}}^{\infty} \mathrm{d} s s^{-q} \\
& \times \int_{0}^{1} \mathrm{~d} \eta\left(1-\eta^{4}\right) J_{1}^{2}\left(s \sqrt{1-\eta^{2}}\right) \frac{1}{\left(1-\eta^{2}\right)^{2} s^{2}+\frac{V_{\mathrm{A}}^{2} R_{\mathrm{L}}^{2}}{\alpha_{\mathrm{F}}^{2}}} .
\end{aligned}
$$

In the last equation, $s=k R_{\mathrm{L}}$, and each term under integration is dimensionless.

\section{Slow mode waves}

With Eqs. (13) and (16), the resonance function (7) for slow mode waves becomes

$$
\mathcal{R}_{\mathrm{S}}^{j}(n)=\frac{2.9 \times 10^{5} \beta V_{\mathrm{A}}^{2} k^{2} \sin ^{2} \theta}{\left.\left(2.9 \times 10^{5} \beta V_{\mathrm{A}}^{2} k^{2} \sin ^{2} \theta\right)^{2}+\left[k v \mu \cos \theta+j V_{\mathrm{A}} k \eta \sqrt{\frac{\beta}{1+\beta}}+n \Omega\right)\right]^{2}} .
$$

The non-vanishing parallel magnetic field component $B_{\|} \neq 0$ (see Eq. (14)) of slow mode waves allows TTD interactions with $n=0$, so that we procede with $n=0$. The resonance function (30) becomes

$$
\begin{aligned}
\mathcal{R}_{S}^{j}(0) & =\frac{2.9 \times 10^{5} \beta V_{\mathrm{A}}^{2} k^{2} \sin ^{2} \theta}{\left(2.9 \times 10^{5} \beta V_{\mathrm{A}}^{2} k^{2} \sin ^{2} \theta\right)^{2}+\left[k v \mu \cos \theta+j V_{\mathrm{A}} k \eta \sqrt{\frac{\beta}{1+\beta}}\right]^{2}} \\
& =\frac{\alpha_{\mathrm{F}} \sin ^{2} \theta}{\left(\alpha_{\mathrm{F}} k \sin ^{2} \theta\right)^{2}+\left[v \mu \cos \theta+j V_{\mathrm{A}} \eta \sqrt{\frac{\beta}{1+\beta}}\right]^{2}},
\end{aligned}
$$

where $\alpha_{\mathrm{F}}$ is the same as in the fast mode case.

Following the same procedure for fast mode waves in Sect. 3, we assume (20), (22), (23), and imply (24). We derive

$$
\begin{aligned}
D_{\mu \mu}^{\mathrm{S}} \simeq & \frac{\Omega^{2}}{4 B_{0}^{2}}\left(1-\mu^{2}\right) \sum_{j= \pm 1} \int_{-\infty}^{\infty} \mathrm{d} k \int_{-1}^{1} \mathrm{~d} \eta \mathcal{R}_{S}^{j}(0) g^{j}(k) J_{1}^{2}(W) \\
& \times\left[\left(1+\eta^{2}\right)\left(1+\mu^{2} \epsilon^{2} \eta^{2} \frac{\beta}{1+\beta}\right)-4 \mu j \epsilon \eta \sqrt{\frac{\beta}{1+\beta}}\right]
\end{aligned}
$$

which can be simplified if we consider the energetic cosmic ray particles $v \gg V_{\mathrm{A}}$, or namely, the last two terms, to be small (order of $\epsilon$ and $\epsilon^{2}$ ) and neglected. Then, we obtain the same expression as in the case for fast mode waves:

$$
\begin{aligned}
& D_{\mu \mu}^{\mathrm{S}} \simeq \frac{\Omega^{2}}{4 B_{0}^{2}}(q-1)(\delta B)^{2} k_{\min }^{q-1}\left(1-\mu^{2}\right) \int_{k_{\min }}^{k_{\max }} \mathrm{d} k k^{-q} \\
& \times \int_{-1}^{1} \mathrm{~d} \eta \mathcal{R}_{\mathrm{S}}(0) J_{1}^{2}(W)\left(1+\eta^{2}\right) .
\end{aligned}
$$

Next, we have to approximate the resonance function for slow mode waves. As in the fast mode case, there are two cases:

$$
\begin{aligned}
& \text { a) } \eta<\eta_{\mathrm{c}}^{\mathrm{S}} \\
& \text { b) } \eta>\eta_{\mathrm{c}}^{\mathrm{s}}
\end{aligned}
$$

where

$\eta_{\mathrm{c}}^{\mathrm{S}}=\frac{\epsilon}{\mu} \frac{\beta}{1+\beta}$.

Note that $\eta_{\mathrm{c}}^{\mathrm{S}}<\eta_{\mathrm{c}}^{\mathrm{F}}$. Using $D_{\mu \mu}^{\mathrm{S}}(-\mu)=D_{\mu \mu}^{\mathrm{S}}(\mu)$ and the substitution $s=R_{\mathrm{L}} k \sqrt{1-\mu^{2}}$, we derive

$D_{\mu \mu}^{\mathrm{S}} \simeq \alpha_{\mathrm{F}}(q-1)\left(k_{\min } R_{\mathrm{L}}\right)^{q-1}\left(\frac{\delta B}{B_{0}}\right)^{2}\left(1-\mu^{2}\right)^{\frac{q+1}{2}}$
$\times \int_{k_{\min }}^{\infty} R_{\mathrm{L}_{\sqrt{1-\mu^{2}}}} \mathrm{~d} s s^{-q}\left[\int_{0}^{\min \left(1, \frac{\epsilon}{\mu} \frac{\beta}{1+\beta}\right)} \mathrm{d} \eta\left(1-\eta^{4}\right)\right.$

$\times J_{1}^{2}\left(s \sqrt{1-\eta^{2}}\right) \frac{1}{\frac{\alpha_{\mathrm{F}}^{2}\left(1-\eta^{2}\right)^{2} s^{2}}{R_{\mathrm{L}}^{2}\left(1-\mu^{2}\right)}+V_{\mathrm{A}}^{2} \eta^{2} \frac{\beta}{1+\beta}}+\int_{\min \left(1, \frac{\epsilon}{\mu} \frac{\beta}{1+\beta}\right)}^{1}$

$\left.\times \mathrm{d} \eta\left(1-\eta^{4}\right) J_{1}^{2}\left(s \sqrt{1-\eta^{2}}\right) \frac{1}{\frac{\alpha_{\mathrm{F}}^{2}\left(1-\eta^{2}\right)^{2} s^{2}}{R_{\mathrm{L}}^{2}\left(1-\mu^{2}\right)}+(v \mu \eta)^{2}}\right]$,

where $\epsilon=V_{\mathrm{A}} / v$.

\subsection{High values of $\mu>\epsilon$}

For large pitch-angles $\mu>\epsilon$ we obtain

$$
\begin{aligned}
D_{\mu \mu}^{\mathrm{S}}(\mu>\epsilon) \simeq & \frac{(q-1)}{\alpha_{\mathrm{F}}}\left(k_{\min } R_{\mathrm{L}}\right)^{q-1}\left(\frac{\delta B}{B_{0}}\right)^{2}\left(1-\mu^{2}\right)^{\frac{q+3}{2}} \\
& \times \int_{k_{\min }} R_{\mathrm{L}} \sqrt{1-\mu^{2}} \mathrm{~d} s s^{-q} \\
& \times\left[\int_{0}^{\frac{\epsilon \beta}{\mu(1+\beta)}} \mathrm{d} \eta\left(1-\eta^{4}\right) J_{1}^{2}\left(s \sqrt{1-\eta^{2}}\right)\right. \\
& \times \frac{1}{\left(1-\eta^{2}\right)^{2} s^{2}+\frac{R_{\mathrm{L}}^{2}\left(1-\mu^{2}\right) V_{\mathrm{A}}^{2} \eta^{2} \frac{\beta}{1+\beta}}{\alpha_{\mathrm{F}}^{2}}} \\
& +\int_{\frac{\epsilon \beta}{\mu(1+\beta)}}^{1} \mathrm{~d} \eta\left(1-\eta^{4}\right) J_{1}^{2}\left(s \sqrt{1-\eta^{2}}\right) \\
& \left.\times \frac{1}{\left(1-\eta^{2}\right)^{2} s^{2}+\frac{R_{\mathrm{L}}^{2}\left(1-\mu^{2}\right)(v \mu \eta)^{2}}{\alpha_{\mathrm{F}}^{2}}}\right]
\end{aligned}
$$




\subsection{Low values of $\mu<\epsilon$}

This case is important for treating damped waves for the same reason as discussed for fast mode waves (Sect. 3). For the small pitch-angles $\mu<\epsilon$, we obtain

$$
\begin{aligned}
& D_{\mu \mu}^{\mathrm{S}}(\mu<\epsilon) \simeq \alpha_{\mathrm{F}}(q-1)\left(k_{\min } R_{\mathrm{L}}\right)^{q-1}\left(\frac{\delta B}{B_{0}}\right)^{2}\left(1-\mu^{2}\right)^{\frac{q+1}{2}} \\
\times & \int_{k_{\min }} R_{\mathrm{L}} \sqrt{1-\mu^{2}} \mathrm{~d} s s^{-q} \\
\times & {\left[\int_{0}^{1} \mathrm{~d} \eta\left(1-\eta^{4}\right) J_{1}^{2}\left(s \sqrt{1-\eta^{2}}\right) \frac{1}{\frac{\alpha_{\mathrm{F}}^{2}\left(1-\eta^{2}\right)^{2} s^{2}}{R_{\mathrm{L}}^{2}\left(1-\mu^{2}\right)}+V_{\mathrm{A}}^{2} \eta^{2} \frac{\beta}{1+\beta}}\right], }
\end{aligned}
$$

or for $\mu=0$, we read

$$
\begin{aligned}
& D_{\mu \mu}^{\mathrm{S}}(\mu=0) \simeq \frac{\Omega^{2}(q-1) R_{\mathrm{L}}^{2}}{4 \alpha_{\mathrm{F}}}\left(k_{\min } R_{\mathrm{L}}\right)^{q-1}\left(\frac{\delta B}{B_{0}}\right)^{2} \int_{k_{\min }} R_{\mathrm{L}}^{\infty} \mathrm{d} s s^{-q} \\
& {\left[\int_{0}^{1} \mathrm{~d} \eta\left(1-\eta^{4}\right) J_{1}^{2}\left(s \sqrt{1-\eta^{2}}\right) \frac{1}{\left(1-\eta^{2}\right)^{2} s^{2}+\frac{V_{\mathrm{A}}^{2} \eta^{2} \frac{\beta}{1+\beta} R_{\mathrm{L}}^{2}}{\alpha_{\mathrm{F}}^{2}}}\right] .}
\end{aligned}
$$

In the last equation, $s=k R_{\mathrm{L}}$.

\section{Cosmic ray mean free path}

\subsection{Fast mode waves}

In this section, we calculate the mean free path, which is connected with the spatial diffusion coefficient through

$\lambda=\frac{3 \kappa}{v}=\frac{3 v}{4} \int_{0}^{1} \mathrm{~d} \mu \frac{\left(1-\mu^{2}\right)^{2}}{D_{\mu \mu}}$.

For the case in which we are interested, we can write

$$
\begin{aligned}
\lambda^{0 \mathrm{~F}} & =\frac{3 \kappa}{v}=\frac{3 v}{4} \frac{1}{D_{\mu \mu}(\mu=0)} \int_{0}^{\epsilon} \mathrm{d} \mu=\frac{3}{4} \frac{V_{\mathrm{A}}}{D_{\mu \mu}^{F}(\mu=0)} \\
& =3 \frac{\alpha_{\mathrm{F}}}{(q-1) V_{\mathrm{A}}}\left(k_{\min } R_{\mathrm{L}}\right)^{1-q}\left(\frac{B_{0}}{\delta B}\right)^{2} \frac{1}{G},
\end{aligned}
$$

where

$$
\begin{aligned}
G= & \int_{k_{\min } R_{\mathrm{L}}}^{\infty} \mathrm{d} s s^{-q} \int_{0}^{1} \mathrm{~d} \eta\left(1-\eta^{4}\right) J_{1}^{2}\left(s \sqrt{1-\eta^{2}}\right) \\
& \times \frac{1}{\left(1-\eta^{2}\right)^{2} s^{2}+\frac{V_{\mathrm{A}}^{2} R_{\mathrm{L}}^{2}}{\alpha_{\mathrm{F}}^{2}}} .
\end{aligned}
$$

Now, we consider two limits: $k_{\min } R_{\mathrm{L}} \ll 1$, and $k_{\min } R_{\mathrm{L}} \gg 1$, where $k_{\min } R_{\mathrm{L}}=T=E$ and is normalized with respect to $E_{\mathrm{c}}=T_{\mathrm{c}}=\frac{k_{\mathrm{c}}}{k_{\min }} m_{\mathrm{e}} c^{2}$. Expressing $k_{\min }=L_{\max } / 2 \pi$ in the terms of the longest wavelength of isotropic fast mode waves, $k_{\mathrm{c}}=$ $\Omega_{0, \mathrm{e}} / v_{\mathrm{A}}=\omega_{\mathrm{p}, \mathrm{e}} / c$ and for following plasma conditions $\left(v_{\mathrm{A}}=\right.$ $33.5 \frac{\mathrm{km}}{\mathrm{s}}, \frac{B_{0}}{\delta B}=1$, Beck 2007), $\left.T_{\mathrm{c}}=10.7 n_{\mathrm{e}}^{1 / 2}\left(\frac{L_{\max }}{10 \mathrm{pc}}\right) \times 10^{9} \mathrm{MV}\right)$. For these values, the particle mean free path is measured by $\lambda_{1}=\frac{9 \alpha_{\mathrm{F}}}{V_{\mathrm{A}}}\left(\frac{B_{0}}{\delta B}\right)^{2}$. We have used interplanetary plasma conditions that have been used in both models (Schlickeiser et al. 2010; Teufel \& Schlickeiser 2002) for comparison. We note that for interstellar medium plasma conditions, $\lambda_{1}$ is one or two orders of magnitude larger than for interplanetary conditions, which is also a reasonable value.
For $k_{\min } R_{\mathrm{L}} \gg 1$ :

This case is treated in detail in Appendix A, where we derive

$G(T \gg 1)=\frac{2}{5} \frac{10^{-14}}{q} T^{-(q+2)}$,

$\lambda^{0 \mathrm{~F}}(T \gg 1)=\frac{15 \alpha_{\mathrm{F}}}{V_{\mathrm{A}}} \frac{q}{q-1}\left(\frac{B_{0}}{\delta B}\right)^{2} 10^{14} T^{3}$.

At relativistic rigidities, we find that $\lambda^{0} \sim T^{3}$. As we consider certain positron energies $1-100 \mathrm{GeV}$, this case is not relevant. For $k_{\min } R_{\mathrm{L}} \ll 1$ :

This case is treated in detail in Appendix A, where we derive

$G(T \ll 1)=\frac{1}{3} \frac{1}{q-1} T^{1-q}$,

$\lambda^{\mathrm{F} 0}(T \ll 1)=\frac{9 \alpha_{\mathrm{F}}}{V_{\mathrm{A}}}\left(\frac{B_{0}}{\delta B}\right)^{2}$.

In this energy limit, the mean free path is constant with respect to $T$, which is relevant for considered positron energies.

\subsection{Slow mode waves}

In this subsection, we calculate the mean free path which is connected with the spatial diffusion coefficient through

$\lambda^{\mathrm{S}}=\frac{3 \kappa^{\mathrm{S}}}{v}=\frac{3 v}{4} \int_{0}^{1} \mathrm{~d} \mu \frac{\left(1-\mu^{2}\right)^{2}}{D_{\mu \mu}^{\mathrm{S}}}$.

For the case for which we are interested, we can write

$$
\begin{aligned}
\lambda^{\mathrm{S} 0} & =\frac{3 \kappa^{\mathrm{S}}}{v}=\frac{3 v}{4} \frac{1}{D_{\mu \mu}^{\mathrm{S}}(\mu=0)} \int_{0}^{\epsilon} \mathrm{d} \mu=\frac{3}{4} \frac{V_{\mathrm{A}}}{D_{\mu \mu}(\mu=0)} \\
& =3 \frac{\alpha_{\mathrm{F}}}{(q-1) V_{\mathrm{A}}}\left(k_{\min } R_{\mathrm{L}}\right)^{1-q}\left(\frac{B_{0}}{\delta B}\right)^{2} \frac{1}{G},
\end{aligned}
$$

where

$$
\begin{aligned}
& G=\int_{k_{\min } R_{\mathrm{L}}}^{\infty} \mathrm{d} s s^{-q} \int_{0}^{1} \mathrm{~d} \eta\left(1-\eta^{4}\right) J_{1}^{2}\left(s \sqrt{1-\eta^{2}}\right) \\
& \times \frac{1}{\left(1-\eta^{2}\right)^{2} s^{2}+\frac{V_{\mathrm{A}}^{2} \eta^{2} \frac{\beta}{1+\beta} R_{\mathrm{L}}^{2}}{\alpha_{\mathrm{F}}^{2}}} .
\end{aligned}
$$

We consider two limits: $k_{\min } R_{\mathrm{L}} \ll 1$, and $k_{\min } R_{\mathrm{L}} \gg 1$, where $k_{\min } R_{\mathrm{L}}=T=E$ and is normalized with respect to $E_{\mathrm{c}}$.

For $k_{\min } R_{\mathrm{L}} \gg 1$ :

This case is treated in detail in Appendix B, where we derive

$$
\begin{aligned}
G(T \gg 1)= & \frac{\sqrt{2}}{\pi} \frac{|b /(b-1)|+|\log (1-b)|}{q p^{2}} T^{-(q+2),} \\
& \lambda^{0 \mathrm{~S}}(T \gg 1)=\frac{3 \alpha_{\mathrm{F}}}{\sqrt{2} V_{\mathrm{A}}} \frac{q}{(q-1)}\left(\frac{B_{0}}{\delta B}\right)^{2} \\
& \times \frac{\pi p^{2}}{|b /(b-1)|+|\log (1-b)|} T^{3}
\end{aligned}
$$

where $T=k_{\min } R_{\mathrm{L}}$ and $b \ll 1$.

At relativistic rigidities, we find that $\lambda^{0 \mathrm{~S}} \sim T^{3}$.

For $k_{\min } R_{\mathrm{L}} \ll 1$ :

This case is treated in detail in Appendix B, where we derive

$$
\begin{aligned}
& G(T \ll 1)=\frac{1}{3} \frac{1}{q-1} T^{1-q}, \\
& \lambda^{0 S}(T \ll 1)=\frac{9 \alpha_{\mathrm{F}}}{V_{\mathrm{A}}}\left(\frac{B_{0}}{\delta B}\right)^{2} .
\end{aligned}
$$


In this energy domain, the mean free path is constant with respect to $T$, which is important for cosmic ray positrons with Lorentz factor $\gamma<m_{\mathrm{p}} / m_{\mathrm{e}}=1836$. Although the mean free path for slow magnetosonic waves has the same expression as in the case for fast waves for energies less than $T_{\mathrm{c}}$, the low energy limit here is caused by plasma- $\beta$ (Appendix $\mathrm{B}$ ). However, it is always less than $T_{\mathrm{c}}$ and does not violate previous conditions.

The turbulence model considered in this paper ensures that all energy particles below the $T_{\mathrm{c}}$ value are scattered by the proposed resonant interaction.

Considering dynamical magnetic slab turbulence and random sweeping slab turbulence (Teufel \& Schlickeiser 2002), there is a sharp cutoff of the turbulence power spectrum at $k_{\min }$. The consequence is that the mean free path for all particles with energies higher than $10^{10} \mathrm{eV}$ with typical parameters rapidly grows and becomes larger than the size of ambient interstellar medium. For the slab plasma waves model (Schlickeiser et al. 2010) at rigidities less than $2 \times 10^{8} \mathrm{eV}$, the positron mean free path becomes infinitely large. It is because these positrons find no LH polarized slab waves with which to resonantly interact. In the turbulence model proposed in this paper, the positron mean free path does not depend on energy, in domain from $10^{5} \mathrm{eV}$ to $10^{12} \mathrm{eV}$.

The comparison of our result with previously reported turbulence models are given in Fig. 1, where the mean free path is in units of $\lambda_{1}=0.2 \beta \mathrm{AU}\left(\mathrm{AU}=1.5 \times 10^{13} \mathrm{~cm}\right)$. To interpret the $\mathrm{MeV}$ positrons propagation and explain their diffusion, the model of the dynamical turbulence can be applied. However, this model fails to explain transport mechanisms for energetic positrons with energies of $100 \mathrm{GeV}$. In contrast, the slab gyroresonat plasma wave turbulence model is able to hold on energetic $\mathrm{GeV}$ positrons but fails in explaining the transport properties of $\mathrm{MeV}$ positrons. The isotropic damped magnetosonic plasma wave turbulence model, which is proposed in this paper, seems to be more plausible, since it covers energies in both domains. This model could be used in modeling turbulence that scatters positrons, resolving the problems stated by the results of either the INTEGRAL or PAMELA experiments.

The inclusion of viscous damping implies either steep turbulence spectra $(q>2.4)$ for isotropic turbulence with fixed values of $k_{\max }$ and $k_{\min }$, or the existence of high wavenumber cutoff for isotropic turbulence with a spectral index $q=$ $5 / 3$ (Spanier \& Schlickeiser 2005). To justify the use of $k_{\max }$ and $k_{\min }$ in our model, it is necessary to assume turbulence with a spectral index that is not less than 2.4. This value is higher than the Kolmogorov value of 5/3 (Kolmogorov 1941) or Kraichan-Iroshnikov value of 3/2 (Kraichnan 1965), but both theories consider an inertial range, where damping effects have been neglected. Interstellar medium has strong damping features, which does not mean that there is no inertial range. It could be that there is an intermediate regime between the inertial and dissipation range. Thus, the request for steep turbulence spectra is not in contradiction to neither Kolmogorov theory, or our calculation, in which there is only a restriction on $\mathrm{q}$ to be greater than 1 .

The broadening of the resonance function could also be insured by nonlinear treatment of the specific turbulence model. Nonlinear theory (second order theory of Shalchi 2005) applied to the magnetostatic slab model (Shalchi et al. 2009) would lead to a broadened resonance function that is similar to the one obtained for the random sweeping model, which is linear (Teufel $\&$ Schlickeiser 2002). We have already mentioned that this linear model could be used to explain $\mathrm{MeV}$ positrons but fails for $\mathrm{GeV}$ positrons. Thus, we expect, at least mathematically, that

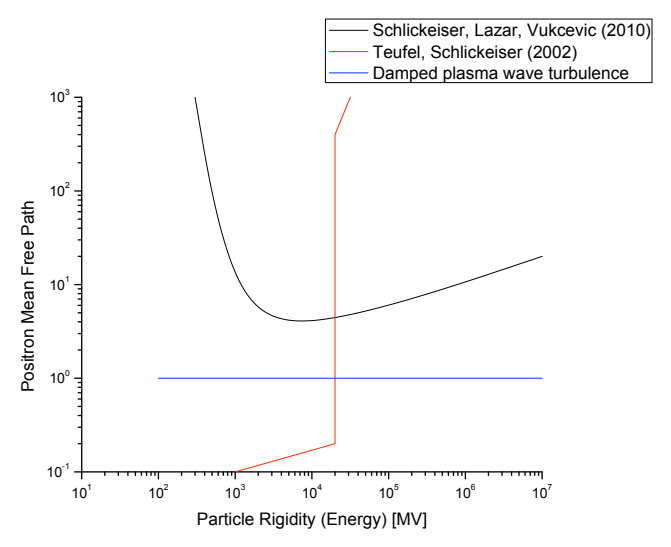

Fig. 1. The positron mean free path. The black line represents the mean free path obtained using the slab turbulence model and gyroresonant interactions, which exhibit a cutoff at particle energies less than $2 \times 10^{8} \mathrm{eV}$. The red line is the mean free path obtained using the dynamical magnetic slab turbulence, or the random sweeping slab turbulence model, which exhibit a sharp cutoff at particle energies of $10^{10} \mathrm{eV}$. The blue line is the mean free path obtained using isotropic magnetosonic damped plasma wave turbulence, which remains constant for particle energies from $10^{5} \mathrm{eV}$ to $10^{12} \mathrm{eV}$. All mean free paths are normalized with respect to the $\lambda_{1}$ value.

inclusion of nonlinear effects to the slab model would have the cutoff at the same wavenumbers as in the random sweeping linear model. However, it would be useful to compare timescales of nonlinear effects and damping effects within the same turbulence model, in order to make a general conclusion on the importance of each of them.

\section{Summary and conclusion}

We have investigated the implications of isotropically distributed interstellar damped plasma waves on the scattering mean free path on the cosmic ray positrons with a Lorentz factor $\gamma<$ $m_{\mathrm{p}} / m_{\mathrm{e}}=1836$. We show that inclusion of resonance broadening due to wave damping in the resonance function guarantees that dominance of transit-time damping also holds for cosmic ray particles at small pitch angle cosines $\mu \leq\left|V_{\mathrm{a}} / v\right|$, unlike the case of negligible wave damping.

For small rigidities, or consequently low energies $T<T_{\mathrm{c}}$, the mean free path is constant with respect to energy. The mean free path at high energies $T>T_{\mathrm{c}}$ approaches a much steeper dependence, namely $\lambda \sim T^{3}$ for both fast and slow waves.

It is difficult to draw any general conclusion on the ratio of fast and slow modes mean free path, since there is an integral dependence on the pitch angle and the plasma-beta parameter are mixed in the latter case. For cold plasma, the mean free path of slow mode merges to the mean free path of the fast one, as expected. However, this turbulence model is able to ensure scattering of the cosmic ray positrons with Lorentz factor $\gamma<m_{\mathrm{p}} / m_{\mathrm{e}}=1836$ via resonant interaction.

\section{Appendix A: Evaluation of the function $G$ for fast mode waves}

The task is to calculate the function $\mathrm{G}(41)$ :

$$
\begin{aligned}
G & =\int_{k_{\min } R_{\mathrm{L}}}^{\infty} \mathrm{d} s s^{-q} \int_{0}^{1} \mathrm{~d} \eta\left(1-\eta^{4}\right) J_{1}^{2}\left(s \sqrt{1-\eta^{2}}\right) \\
& \times \frac{1}{\left(1-\eta^{2}\right)^{2} s^{2}+\frac{V_{\mathrm{A}}^{2} R_{\mathrm{L}}^{2}}{\alpha_{\mathrm{F}}^{2}}} .
\end{aligned}
$$


First, it is possible to evaluate the term $\frac{V_{\mathrm{A}}^{2} R_{\mathrm{L}}^{2}}{\alpha_{\mathrm{F}}^{2}} \sim 10^{14}$ for used plasma parameters (Sect. 5). We then evaluate $G$ in two energy limits.

\section{Case $G\left(k_{\min } R_{\mathrm{L}} \gg 1\right)$}

For energies $T \gg 1$ we substitute $s=x T$. Then, (A.1) reads as

$$
\begin{aligned}
G & =\int_{1}^{\infty} \mathrm{d} x x^{-q} T^{-(1+q)} \int_{0}^{1} \mathrm{~d} \eta\left(1-\eta^{4}\right) J_{1}^{2}\left(x T \sqrt{1-\eta^{2}}\right) \\
& \times \frac{1}{\left(1-\eta^{2}\right)^{2} x^{2}+10^{14}}=T^{-(1+q)} \int_{1}^{\infty} \mathrm{d} x x^{-q} \\
& \times\left(\int_{0}^{1-\frac{1}{2 x T}} \mathrm{~d} \eta\left(1-\eta^{4}\right)\right) \frac{1}{\pi x T \sqrt{1-\eta^{2}}} \frac{1}{\left(1-\eta^{2}\right)^{2} x^{2}+10^{14}} \\
& +\int_{1-\frac{1}{2 x T}}^{1} \mathrm{~d} \eta\left(1-\eta^{4}\right) \frac{1}{4} x^{2} T^{2}\left(1-\eta^{2}\right) \\
& \left.\times \frac{1}{\left(1-\eta^{2}\right)^{2} x^{2}+10^{14}}\right)
\end{aligned}
$$

where we use the approximations of Bessel functions for large and small arguments (Abramowitz \& Stegun 1972):

$J_{v}(z \gg 1) \approx \sqrt{\frac{2}{\pi v z}} \cos \left(v z-\frac{(2 v+1) \pi}{4}\right)$,

implying

$$
\begin{aligned}
J_{1}^{2}(z \gg 1) & =\frac{1}{\pi x T \sqrt{1-\eta^{2}}}\left(1-\sin \left(2 x T \sqrt{1-\eta^{2}}\right)\right) \\
& \simeq \frac{1}{\pi x T \sqrt{1-\eta^{2}}}
\end{aligned}
$$

$(1 / \xi \gg \sin \xi / \xi)$ for the argument, $z=x T \ll 1$, and

$J_{v}(z \ll 1) \approx \frac{(z / 2)^{v}}{\Gamma(v+1)}$,

implying

$J_{1}^{2}(z \ll 1)=\frac{1}{4} x^{2} T^{2}\left(1-\eta^{2}\right)$

for the argument $z=x T \gg 1$. We then obtain

$T^{(1+q)} G(T \gg 1)=\frac{T^{2}}{4} \int_{1}^{\infty} \mathrm{d} x x^{-q+2} \int_{1-\frac{1}{2 x T}}^{1} \mathrm{~d} \eta\left(1-\eta^{4}\right)$

$\frac{\left(1-\eta^{2}\right)}{\left(1-\eta^{2}\right)^{2} x^{2}+10^{14}}$

$+\frac{1}{\pi T} \int_{1}^{\infty} \mathrm{d} x x^{-(q+1)} \int_{0}^{1} \mathrm{~d} \eta \frac{\left(1-\eta^{4}\right)}{\sqrt{1-\eta^{2}}} \frac{1}{\left(1-\eta^{2}\right)^{2} x^{2}+10^{14}}$

$-\frac{1}{\pi T} \int_{1}^{\infty} \mathrm{d} x x^{-(q+1)} \int_{1-\frac{1}{2 x T}}^{1} \mathrm{~d} \eta \frac{\left(1-\eta^{4}\right)}{\sqrt{1-\eta^{2}}} \frac{1}{\left(1-\eta^{2}\right)^{2} x^{2}+10^{14}}=$

$I_{3}+I_{1}-I_{2}$.

Next, we simplify further, namely keeping only terms with respect to $1-\eta$ of the lowest order, and we evaluate each integral:

$$
\begin{aligned}
I_{2} & =\frac{1}{\pi T} \int_{1}^{\infty} \mathrm{d} x x^{-(q+1)} \int_{0}^{\frac{1}{2 x T}} \mathrm{~d} m \frac{4 m}{\sqrt{2 m}} \frac{1}{4 m^{2} x^{2}+10^{14}} \\
& =\frac{2}{3} \frac{10^{-14}}{\pi(q+3 / 2)} T^{-\frac{5}{2}},
\end{aligned}
$$

where we substitute $m=1-\eta$.

$I_{3}=\frac{T^{2}}{4} \int_{1}^{\infty} \mathrm{d} x x^{-q+2} \int_{0}^{\frac{1}{2 x T}} \mathrm{~d} m 4 m \frac{2 m}{10^{14}}==\frac{1}{12} \frac{10^{-14}}{q} T^{-1}$,

where we have used the same substitution as in the previous case and where $10^{14} \gg 4 m^{2} x^{2}$. We note that $I_{2} \ll I_{3}$.

$I_{1}=\frac{1}{\pi T} \int_{1}^{\infty} \mathrm{d} x x^{-(q+1)} \int_{0}^{1} \mathrm{~d} \eta \frac{\left(1-\eta^{4}\right)}{\sqrt{1-\eta^{2}}} \frac{1}{10^{14}}=\frac{5}{16} \frac{10^{-14}}{q} \frac{1}{T}$.

Combining all these three integrals, we obtain

$G(T \gg 1)=\frac{2}{5} \frac{10^{-14}}{q} T^{-(q+2)}$.

Case $\mathrm{G}\left(k_{\min } R_{\mathrm{L}} \ll 1\right)$

For energies $T \ll 1$, we use the approximation for Bessel function (A.5). Then, (A.1) reads as

$$
\begin{aligned}
G(T \ll 1) & =\frac{1}{4} \int_{k_{\min } R_{\mathrm{L}}}^{1} \mathrm{~d} s s^{-q} \int_{0}^{1} \mathrm{~d} \eta\left(1-\eta^{4}\right) \frac{\left(1-\eta^{2}\right) s^{2}}{\left(1-\eta^{2}\right)^{2} s^{2}+\frac{V_{\mathrm{A}}^{2} R_{\mathrm{L}}^{2}}{\alpha_{\mathrm{F}}^{2}}} \\
& =\frac{1}{4} \int_{T}^{1} \mathrm{~d} s s^{-q} \int_{0}^{1} \mathrm{~d} \eta\left(1+\eta^{2}\right)-\frac{M^{2}}{4} \\
& \times \int_{T}^{1} \mathrm{~d} s s^{-q} \int_{0}^{1} \mathrm{~d} \eta\left(1+\eta^{2}\right) \\
& \times \frac{1}{\left(1-\eta^{2}\right)^{2} s^{2}+M^{2}}=I_{5}-I_{4},
\end{aligned}
$$

where $M^{2}=\frac{V_{\mathrm{A}}^{2} R_{\mathrm{L}}^{2}}{\alpha_{\mathrm{F}}^{2}}$. We then evaluate each integral:

$$
\begin{aligned}
I_{5} & =\frac{1}{4} \int_{T}^{1} \mathrm{~d} s s^{-q} \int_{0}^{1} \mathrm{~d} \eta\left(1+\eta^{2}\right)=\frac{1}{3(q-1)}\left(T^{1-q}-1\right) \\
& \simeq \frac{1}{3(q-1)} T^{1-q},
\end{aligned}
$$

since $T \ll 1$, and $q>1$.

$$
\begin{aligned}
I_{4} & =\frac{M^{2}}{4} \int_{T}^{1} \mathrm{~d} s s^{-q} \int_{0}^{1} \mathrm{~d} \eta\left(1+\eta^{2}\right) \frac{1}{\left(1-\eta^{2}\right)^{2} s^{2}+M^{2}} \\
& =\frac{1}{4} \int_{T}^{1} \mathrm{~d} s s^{-q} I_{6},
\end{aligned}
$$

where

$$
I_{6}=\int_{0}^{1} \mathrm{~d} \eta\left(1+\eta^{2}\right) \frac{1}{\left(\left(1-\eta^{2}\right)^{2} s^{2} / M^{2}\right)+1} .
$$

The exact solution of integral $I_{6}$ reads as

$$
\frac{(-1)^{1 / 4}\left(\frac{(-2 \mathrm{i}+n) \arctan \left(\frac{(-1)^{1 / 4}}{\sqrt{-i+n}}\right)}{\sqrt{-i+n}}+\frac{i(2 i+n) \arctan \left(\frac{(-1)^{3 / 4}}{\sqrt{-i+n}}\right)}{\sqrt{-i+n}}\right)}{2 n \sqrt{1+n^{2}}},
$$

A111, page 8 of 10 
M. Vukcevic: The scattering mean free path of cosmic ray particles in isotropic damped plasma wave turbulence

where $n^{2}=M^{2} / s^{2}$. We can evaluate $I_{6}$ by

$I_{6} \approx \frac{1}{2 n^{3}}$,

which for $I_{4}$ gives

$I_{4} \approx \frac{1}{8 M^{3}} \frac{1}{4-q}\left(1-T^{(4-q)}\right)$.

To compare $I_{4}$ and $I_{5}$ and calculate $G(T \ll 1)$, we have to consider two cases, which are $(1<q<4)$ and steep $(4<q<6)$ turbulence spectrum.

For $(1<q<4)$ the integral $I_{4} \sim \frac{1}{4-q}$, while $I_{4} \sim \frac{1}{q-4} T^{q-4}$ for $(4<q<6)$. However, $M^{3}$ exists in both cases in the denominator of $I_{4}$, which implies that $I_{4} \ll I_{5}$. According to Eq. (A.12) then:

$G(T \ll 1)=\frac{1}{3} \frac{1}{q-1} T^{1-q}$.

\section{Appendix B: Evaluation of the function G for slow mode waves}

The task is to calculate the function $\mathrm{G}(48)$,

$$
\begin{aligned}
& G=\int_{k_{\min } R_{\mathrm{L}}}^{\infty} \mathrm{d} s s^{-q} \int_{0}^{1} \mathrm{~d} \eta\left(1-\eta^{4}\right) J_{1}^{2}\left(s \sqrt{1-\eta^{2}}\right) \\
& \frac{1}{\left(1-\eta^{2}\right)^{2} s^{2}+\frac{V_{\mathrm{A}}^{2} \eta^{2} \frac{\beta}{1+\beta} R_{\mathrm{L}}^{2}}{\alpha_{\mathrm{F}}^{2}}} .
\end{aligned}
$$

and evaluate it in two energy limits.

$$
\text { Case } G\left(k_{\min } R_{\mathrm{L}} \gg 1\right)
$$

For energies $T \gg 1$ we substitute $s=x T$. Then, (B.1) reads as

$$
\begin{aligned}
G & =\int_{1}^{\infty} \mathrm{d} x x^{-q} T^{-(1+q)} \int_{0}^{1} \mathrm{~d} \eta\left(1-\eta^{4}\right) J_{1}^{2}\left(x T \sqrt{1-\eta^{2}}\right) \\
& \times \frac{1}{\left(1-\eta^{2}\right)^{2} x^{2}+p^{2} \eta^{2}} \\
= & T^{-(1+q)} \int_{1}^{\infty} \mathrm{d} x x^{-q}\left(\int_{0}^{1-\frac{1}{2 x T}} \mathrm{~d} \eta\left(1-\eta^{4}\right) \frac{1}{\pi x T \sqrt{1-\eta^{2}}}\right. \\
& \times \frac{1}{\left(1-\eta^{2}\right)^{2} x^{2}+p^{2} \eta^{2}} \\
& \left.+\int_{1-\frac{1}{2 x T}}^{1} \mathrm{~d} \eta\left(1-\eta^{4}\right) \frac{1}{4} x^{2} T^{2}\left(1-\eta^{2}\right) \frac{1}{\left(1-\eta^{2}\right)^{2} x^{2}+p^{2} \eta^{2}}\right)
\end{aligned}
$$

where $p^{2}=\left(V_{\mathrm{A}}^{2} R_{\mathrm{L}}^{2} \beta\right) /\left(\alpha_{\mathrm{F}}^{2}(1+\beta)\right)$. We have used

$J_{v}(z \gg 1) \approx \sqrt{\frac{2}{\pi v z}} \cos \left(v z-\frac{(2 v+1) \pi}{4}\right)$,

implying

$$
\begin{aligned}
J_{1}^{2}(z \gg 1) & =\frac{1}{\pi x T \sqrt{1-\eta^{2}}}\left(1-\sin \left(2 x T \sqrt{1-\eta^{2}}\right)\right) \\
& \simeq \frac{1}{\pi x T \sqrt{1-\eta^{2}}}
\end{aligned}
$$

$(1 / \xi \gg \sin \xi / \xi)$ for the argument, $z=x T \ll 1$, and

$J_{v}(z \ll 1) \approx \frac{(z / 2)^{v}}{\Gamma(v+1)}$,

implying

$J_{1}^{2}(z \ll 1)=\frac{1}{4} x^{2} T^{2}\left(1-\eta^{2}\right)$

for the argument $z=x T \gg 1$. We then obtain

$T^{(1+q)} G(T \gg 1)=\frac{T^{2}}{4} \int_{1}^{\infty} \mathrm{d} x x^{-q+2} \int_{1-\frac{1}{2 x T}}^{1} \mathrm{~d} \eta\left(1-\eta^{4}\right)$

$\times \frac{\left(1-\eta^{2}\right)}{\left(1-\eta^{2}\right)^{2} x^{2}+p^{2} \eta^{2}}+\frac{1}{\pi T} \int_{1}^{\infty} \mathrm{d} x x^{-(q+1)}$

$\int_{0}^{1} \mathrm{~d} \eta \frac{\left(1-\eta^{4}\right)}{\sqrt{1-\eta^{2}}} \frac{1}{\left(1-\eta^{2}\right)^{2} x^{2}+p^{2} \eta^{2}}-\frac{1}{\pi T} \int_{1}^{\infty} \mathrm{d} x x^{-(q+1)}$

$\int_{1-\frac{1}{2 x T}}^{1} \mathrm{~d} \eta \frac{\left(1-\eta^{4}\right)}{\sqrt{1-\eta^{2}}} \frac{1}{\left(1-\eta^{2}\right)^{2} x^{2}+p^{2} \eta^{2}}$

$=I_{3}+I_{1}-I_{2}$.

Next, we evaluate each integral in turn as in the case of fast mode waves.

$$
\begin{aligned}
I_{2} & =\frac{1}{\pi T} \int_{1}^{\infty} \mathrm{d} x x^{-(q+1)} \int_{0}^{\frac{1}{2 x T}} \mathrm{~d} m \frac{4 m}{\sqrt{2 m}} \frac{1}{4 m^{2} x^{2}+p^{2}(1-m)^{2}} \\
& =\frac{1}{\pi T} \int_{1}^{\infty} \mathrm{d} x x^{-(q+1)} I_{2}^{\prime}(m, x)
\end{aligned}
$$

where we substitute $m=1-\eta$.

$$
\begin{aligned}
I_{3} & =\frac{T^{2}}{4} \int_{1}^{\infty} \mathrm{d} x x^{-q+2} \int_{0}^{\frac{1}{2 x T}} \mathrm{~d} m \frac{8 m^{2}}{p^{2}(1-m)^{2}+4 m^{2} x^{2}} \\
& =\frac{T^{2}}{4} \int_{1}^{\infty} \mathrm{d} x x^{-q+2} I_{3}^{\prime}(m, x),
\end{aligned}
$$

where we have used the same substitution as in the previous case.

$$
\begin{aligned}
I_{1} & =\frac{1}{\pi T} \int_{1}^{\infty} \mathrm{d} x x^{-(q+1)} \int_{0}^{1} \mathrm{~d} m \frac{4 m}{\sqrt{2 m}} \frac{1}{p^{2}(1-m)^{2}+4 m^{2} x^{2}} \\
& =\frac{1}{\pi T} \int_{1}^{\infty} \mathrm{d} x x^{-(q+1)} I_{1}^{\prime}(m, x) .
\end{aligned}
$$

To compare functions under integration with respect to $m$, we write out the following integrals

$$
\begin{aligned}
I_{2}^{\prime}(m, x) & =\int_{0}^{\frac{1}{2 x T}} \mathrm{~d} m \frac{4 m}{\sqrt{2 m}} \frac{1}{4 m^{2} x^{2}+p^{2}(1-m)^{2}} \\
& =\frac{2 \sqrt{2}}{p^{2}} \int_{0}^{\frac{1}{2 x T}} \mathrm{~d} m f_{1},
\end{aligned}
$$

$I_{3}^{\prime}(m, x)=\int_{0}^{\frac{1}{2 x T}} \mathrm{~d} m \frac{8 m^{2}}{p^{2}(1-m)^{2}+4 m^{2} x^{2}}=\frac{8}{p^{2}} \int_{0}^{\frac{1}{2 x T}} \mathrm{~d} m f_{2}$,

$$
I_{1(m, x)}^{\prime}=\int_{0}^{1} \mathrm{~d} m \frac{4 m}{\sqrt{2 m}} \frac{1}{p^{2}(1-m)^{2}+4 m^{2} x^{2}}=\frac{2 \sqrt{2}}{p^{2}} \int_{0}^{1} \mathrm{~d} m f_{1},
$$


where $f_{1}=\frac{m}{(1-m)^{2}}$ and $f_{2}=\frac{m^{2}}{(1-m)^{2}}$. We have approximated the denominator as $(1-m)^{2}$, as long as $\frac{4 x^{2}}{p^{2}} \ll 1$ holds (which is ensured by consideration of $T \gg 1$ ). Analyzing $f_{1}$ and $f_{2}$ in given intervals of integration we deduce that $I_{2}<I_{3}<I_{1}$. However, this case is not of particular interest, so we make a rough estimation on $G$.

Integral $I_{1}^{\prime}$ diverges for $m=1$, so we integrate to $m=b<1$. Combining all, we obtain

$G(T \gg 1)=\frac{\sqrt{2}}{\pi} \frac{|b /(b-1)|+|\log (1-b)|}{q p^{2}} T^{-(q+2)}$.

\section{Case $\mathrm{G}\left(k_{\min } R_{\mathrm{L}} \ll 1\right)$}

For energies $T \ll 1$, we use the approximation for Bessel function (B.5). Then, (B.1) reads as

$$
\begin{aligned}
& G(T \ll 1)=\frac{1}{4} \int_{k_{\min } R_{\mathrm{L}}}^{1} \mathrm{~d} s s^{-q} \int_{0}^{1} \mathrm{~d} \eta\left(1-\eta^{4}\right) \\
& \times \frac{\left(1-\eta^{2}\right) s^{2}}{\left(1-\eta^{2}\right)^{2} s^{2}+\frac{V_{\mathrm{A}}^{2} R_{\mathrm{L}}^{2} \eta^{2} \beta}{\alpha_{\mathrm{F}}^{2}(1+\beta)}} \\
& =\frac{1}{4} \int_{T}^{1} \mathrm{~d} s s^{-q} \int_{0}^{1} \mathrm{~d} \eta\left(1+\eta^{2}\right)-\frac{p^{2}}{4} \\
& \int_{T}^{1} \mathrm{~d} s s^{-q} \int_{0}^{1} \mathrm{~d} \eta\left(1+\eta^{2}\right) \\
& \times \frac{\eta}{\left(1-\eta^{2}\right)^{2} s^{2}+p^{2} \eta^{2}} \\
& =I_{5}-I_{4},
\end{aligned}
$$

where $p^{2}=\frac{V_{\mathrm{A}}^{2} R_{\mathrm{L}}^{2} \beta}{\alpha_{\mathrm{F}}^{2}(1+\beta)}$. We then evaluate each integral.

$$
\begin{aligned}
& I_{5}=\frac{1}{4} \int_{T}^{1} \mathrm{~d} s s^{-q} \int_{0}^{1} \mathrm{~d} \eta\left(1+\eta^{2}\right)=\frac{1}{3(q-1)}\left(T^{1-q}-1\right) \\
& \simeq \frac{1}{3(q-1)} T^{1-q},
\end{aligned}
$$

since $T \ll 1$.

To estimate $I_{4}$, we write

$$
\begin{aligned}
I_{4} & =\frac{p^{2}}{4} \int_{T}^{1} \mathrm{~d} s s^{-q} \int_{0}^{1} \mathrm{~d} \eta\left(1+\eta^{2}\right) \frac{\eta^{2}}{\left(1-\eta^{2}\right)^{2} s^{2}+p^{2} \eta^{2}} \\
& =\frac{1}{4} \int_{T}^{1} \mathrm{~d} s s^{-q} I_{6},
\end{aligned}
$$

where

$$
I_{6}=\int_{0}^{1} \mathrm{~d} \eta\left(1+\eta^{2}\right) \frac{\eta^{2}}{\left(\left(1-\eta^{2}\right)^{2} w^{2}\right)+\eta^{2}}
$$

and $w^{2}=s^{2} / p^{2}$. Here, we compare functions in integrals $I_{5}$ with respect to $\eta$, namely $f_{1}=\left(1+\eta^{2}\right)$, and in $I_{6}$ namely $f_{2}=(1+$ $\left.\eta^{2}\right) \frac{\eta^{2}}{\left(\left(1-\eta^{2}\right)^{2} w^{2}\right)+\eta^{2}}$.

We find that $I_{5} \gg I_{6}$ for $w^{2} \gg 1$ over the interval [0,1]. For $10^{-14}<w^{2}<1$, we still have $I_{5}>I_{4}$. Only at $w^{2} \leqslant 10^{-14}$, $I_{5}<I_{6}$ (low energy value is caused by this limitation; namely, we consider $T \ll 1)$. However, this treatment is valid as long as $T>\frac{\beta}{1+\beta}$, which is always less than 1 .

As long as $I_{5}$ dominates, we obtain

$G(T \ll 1)=\frac{1}{3} \frac{1}{q-1} T^{1-q}$.

\section{References}

Abdo, A. A., Ackermann, M., Ajello, M., et al. 2009, Phys. Rew. Lett. 102, 1101 Abramowitz, M., \& Stegun, I. A. 1972, Handbook of Mathematical functions 1966

Achterberg, A. 1979, A\&A, 76, 276

Adriani, O., Barbarino, G. C., Bazilevskaya, G. A., et al. 2009, Nature, 458, 607 Agular, M., et al. 2013, Phys. Rew. Lett., 110, 141102

Arvanitaki, A., Dimopoulos, S., Dubovsky, S., et al. 2009, Phys. Rew. D, 80, 55011

Beck, R. 2007, EAS Pub. Ser., 23, 19

Bieber, J. W., Wanner, W., \& Matthaeus, W. M. 1996, J. Geophys. Res., 101, 2511

Boehm, C., Hooper, D., Silk, J., et al. 2004, Phys. Rev. Lett., 92, 1301

Clayton, D. D. 1973, Nature Phys. Sci., 244, 137

Dogan, A., Spanier, F., Vainio, R., \& Schlickeiser, R. 2006, J. Plasma Phys., 72, 419

Fedorenko, V. N. 1992, Sov. Sci. Rev. E, 8, 4

Guessoum, N., Jean, P., \& Prantzos, N. 2006, A\&A, 457, 753

Higdon, J. C., Lingenfelter, R. E., \& Rothschild, R. E. 2009, ApJ, 698, 350

Hooper, D., Stebbins, A., \& Zurek, K. N. 2009, PRD, 79, 103513

Jokippi, J. R. 1966, ApJ 146, 480

Knödlseder, J., Jean, P., Lonjou, V., et al. 2005, A\&A, 441, 513

Kolmogorov, A. N. 1941, Dokl. Akad. Nauk. SSSR, 30, 301

Kraichnan, R. 1965, Phys. Fluids, 8, 1835

Kubo, R. 1957, J. Phys. Soc. Jpn. 12, 570

Matthaeus, W. H., \& Smith, C. W. 1981, Phys. Rev. A 24, 2135

Milne, P. A., Kurfess, J. D., Kinzer, R. L., \& Leising, M. D. 2002, New Astron. Rev., 46, 553

Palmer, I. D. 1982, Rew. Geophys. Space Phys., 20, 335

Pohl, M., \& Eichler, D. 2010, ApJ, 712, L53

Ramaty, R., \& Lingenfelter, R. E. 1979, Nature, 278, 127

Ramaty, R., Stecker, F. W., \& Misra, D. 1970, J. Geophys. Res., 75, 1141

Schlickeiser, R. 2002, Cosmic Ray Astrophysics (Berlin: Springer)

Schlickeiser, R. 2003, Lect. Not. Phys., 612, 230

Schlickeiser, R., \& Miller, J. A. 1998, ApJ 492, 352

Schlickeiser, R., \& Vainio, R. 1999, Astrophys. Space Sci., 264, 457

Schlickeiser, R., Lazar, M., \& Vukcevic, M. 2010, ApJ, 719, 1497

Shalchi, A. 2005, Phys. Plasmas, 12, 052905

Shalchi, A., \& Schlickeiser, R. 2004, A\&A, 420, 821

Shalchi, A., Škoda, T., Tautz, R. C., \& Schlickeiser, R. 2009, Phys. Rev. D, 80, 023012

Spanier, F., \& Schlickeiser, R. 2005, A\&A, 436, 1

Sturrock, P. A. 1971, ApJ, 164, 529

Teufel, A., \& Schlickeiser, R. 2002, A\&A, 393, 703

Teufel, A., Lerche, I., \& Schlickeiser, R. 2003, A\&A 397, 776

Vukcevic, M., \& Schlickeiser, R. 2007, A\&A, 467, 15 